\title{
REGION-BASED BUILDING ROOFTOP EXTRACTION AND CHANGE DETECTION
}

\author{
J. Tian ${ }^{*}$, L. Metzlaff, P. d’Angelo, P. Reinartz \\ ${ }^{a}$ German Aerospace Center (DLR), Remote Sensing Technology Institute, 82234 Wessling, Germany \\ (Jiaojiao.Tian, Lukas.Metzlaff, Pablo.Angelo, Peter.Reinartz)@dlr.de
}

Commission III, WG III/6

KEY WORDS: Rooftop extraction, change detection, segmentation, DSM

\begin{abstract}
:
Automatic extraction of building changes is important for many applications like disaster monitoring and city planning. Although a lot of research work is available based on 2D as well as 3D data, an improvement in accuracy and efficiency is still needed. The introducing of digital surface models (DSMs) to building change detection has strongly improved the resulting accuracy. In this paper, a post-classification approach is proposed for building change detection using satellite stereo imagery. Firstly, DSMs are generated from satellite stereo imagery and further refined by using a segmentation result obtained from the Sobel gradients of the panchromatic image. Besides the refined DSMs, the panchromatic image and the pansharpened multispectral image are used as input features for mean-shift segmentation. The DSM is used to calculate the nDSM, out of which the initial building candidate regions are extracted. The candidate mask is further refined by morphological filtering and by excluding shadow regions. Following this, all segments that overlap with a building candidate region are determined. A building oriented segments merging procedure is introduced to generate a final building rooftop mask. As the last step, object based change detection is performed by directly comparing the building rooftops extracted from the pre- and after-event imagery and by fusing the change indicators with the rooftop region map. A quantitative and qualitative assessment of the proposed approach is provided by using WorldView-2 satellite data from Istanbul, Turkey.
\end{abstract}

\section{INTRODUCTION}

High and very high resolution remote sensing data consisting of satellite observations of land surface offer new opportunities for timely and accurate change detection, which could be used for understanding the relationships and interactions between human and natural phenomena in order to promote a better decision making. Various change detection technologies based on satellite imagery have been extensively studied and applied. (Singh, 1989; Petit et al., 2001; Coppin et al., 2004; Lu et al., 2004; Liu et al., 2014). Classical 2D image processing techniques are laborious due to the higher amount of features required to locate buildings. This problem can be avoided when 3D information is available.

Besides LiDAR, 3D information can be derived from satellite stereo imagery, which exhibit a larger field of view and are available at a lower cost per $\mathrm{km}^{2}$ with respect to aerial imagery. In recent years, 3D change detection has gained considerable attention as it could distinguish the changes that are not clear in 2D imagery, and it can also avoid some mis-detection caused by differences of the compared images in sensor geometry models and photometric properties (Qin et al., 2016). As one of the important 3D change detection approaches, post-classification methods compare the resulting labels from classification/objects detection results, which avoid direct comparison of spectral or height information. In addition, post-classification methods are able to provide a type change matrix and reuse historic landuse/land-cover results. However, accuracy of these methods mostly depends on the mono-temporal classification /object detection results. Therefore, in the context of rooftop change detection, the rooftop detection approach is of crucial importance to improve the final result.

Although many rooftop/building extraction approaches have been proposed for stereo imagery and still an improvement in robustness and efficiency is required, especially for buildings with complex shapes. An image based method usually starts with pixel based classification. Through clustering/segmentation the accuracy of the classification can be improved. In this paper, an improved segmentation approach is proposed to enhance the quality of the Digital Surface Model (DSM) and to generate a region map for rooftop extraction. In the second procedure, the region map is used to extract the accurate rooftop masks, which are further adopted in post-classification and region-based change detection approaches. In section 3 , the proposed method has been tested on WorldView-2 data which were captured over Istanbul, Turkey. Discussions and conclusions are presented in Section 4 and Section 5.

\section{METHODOLOGY}

To improve the accuracy of building extraction and change detection, a region-based building rooftop extraction and change detection approach is proposed. Firstly the region map is prepared based on a two-step segmentation approach. In the second step the region map is adopted to assist the building rooftop extraction and change detection procedure.

\subsection{Segmentation}

2.1.1 Workflow: As the quality of the DSM has a large impact on the results of further steps, as shown in Figure 1, in the first step, the DSM quality has to be analyzed and improved before adopting it for segmentation. In the mean-shift segmentation procedure the pansharpened multispectral data and the improved DSMs are used as input. 


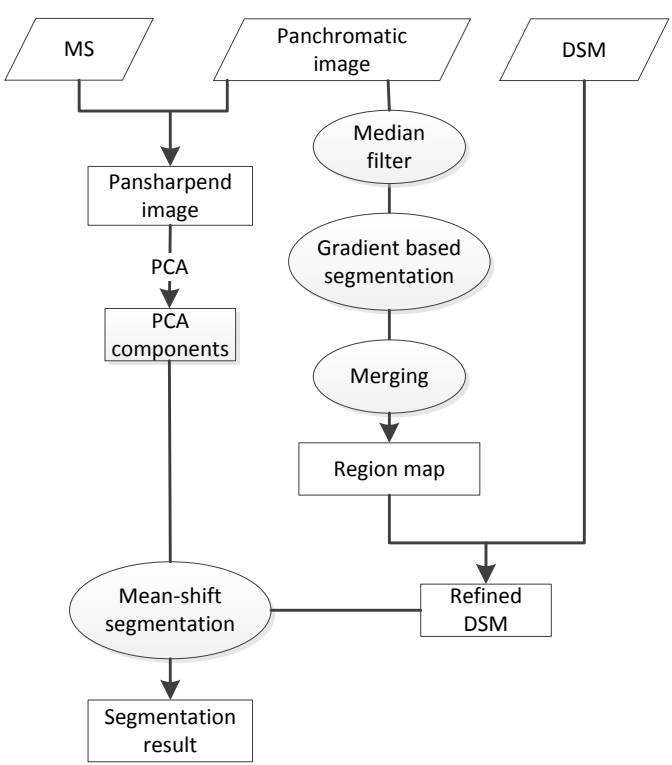

Figure 1. Workflow of the segmentation

\subsubsection{Gradient based segmentation}

As a first step for the gradient based segmentation, noise in the panchromatic image is reduced with a median filter. Subsequently the image contrast is adjusted to provide a better distinction between buildings and their surroundings. Figure 2 shows an example of the prepared panchromatic image (Figure 2a) and the prepared segments (Figure 2d) based on the lines extracted by Sobel gradient (Figure 2c).

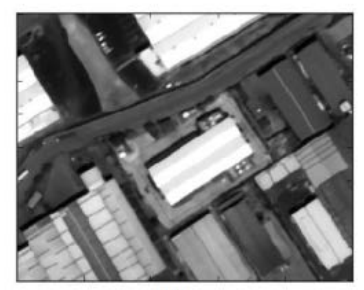

(a)

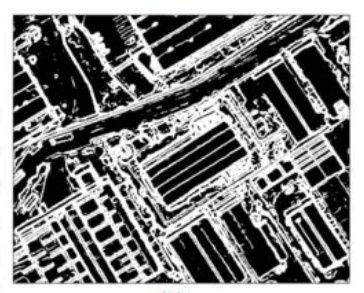

(c)

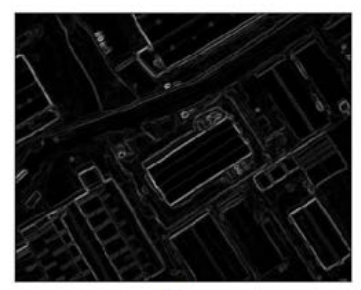

(b)

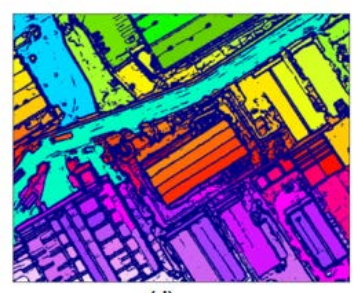

(d)
Figure 2: (a) Original panchromatic image; (b) Sobel gradient in horizontal and vertical direction; (c) Sobel lines (d) Original segment.

\subsubsection{DSM refinement}

The initial region map presented in Figure 2 (d) is an obvious over-segmentation result. Thus a region merging procedure is necessary. Several procedures are adopted for this purpose. Some small sized regions are mainly located at object boundaries. Therefore, these small segments with a size below a predefined threshold are assigned a "no data" - value, zero for instance. In the first step, the small sized regions that are encapsulated by other regions are merged to their neighbours. If more than one neighbour is available, the label of the most similar segment is selected (Tian et al., 2013).
The DSM can then be refined by using the resulted region map. However, one of the possible remaining problems after merging is under-segmentation, which is introduced mainly by similar textures between building roofs and streets. Thus, an error check is performed to avoid introducing inaccurate height values for some large regions. In this approach the average height over the segments are compared to the original height of these pixels. If the mean standard error between this average height and the original height is above a threshold $T$, the segment is not used for the refined DSM. Instead the original height values are kept in the refined DSM.

As shown in Figure 3. Figure 3 (a) is the original DSM that is characterized with unsharpened edges. Figure 3(b) is the refined DSM by directly taking the average height for all regions. Figure 3(c) is the refined DSMs with restriction check.
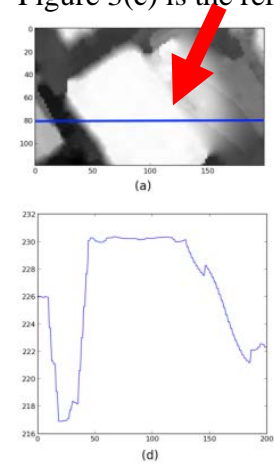
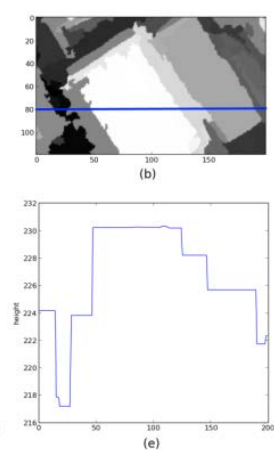
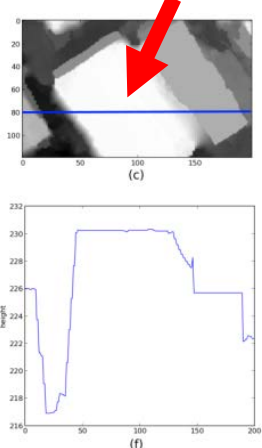

Figure 3. (a) Original DSM. (b) Refined DSM with average over boundaries. (c) Refined DSM result. (d,e,f) Height profiles of a,b,c.

\subsubsection{Region map preparation}

In this section the principal component analysis (PCA), is performed on the pan-sharpened image. The components and the refined DSMs are used in the mean-shift segmentation procedure (Comaniciu and Meer, 2002). After that all segments that are smaller than a predefined size are merged to their neighbour region with the most similar height. Furthermore, encapsulated areas are merged, as they are assumed to be part of the segment that surrounds them. Figure 4 shows the significant reduction of noise in the segmentation result after these two steps.

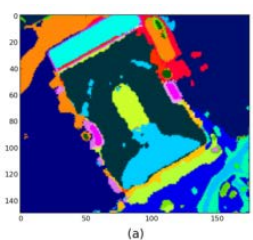

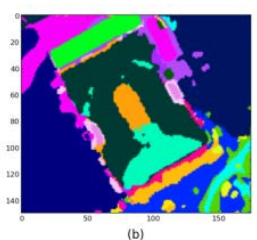

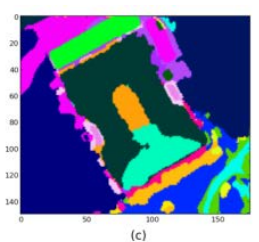

Figure 4: (a) Segmentation result. (b) Segmentation after merging small areas. (c) Segmentation after merging based on encapsulation.

\subsection{Building mask generation and change detection}

The workflow of the second part of the proposed approach is shown in Figure 5 . At first the initial building candidate segments are determined, after that a building oriented merging is performed. As the last step change detection can be performed based on the building masks derived from the preand after-event datasets. 


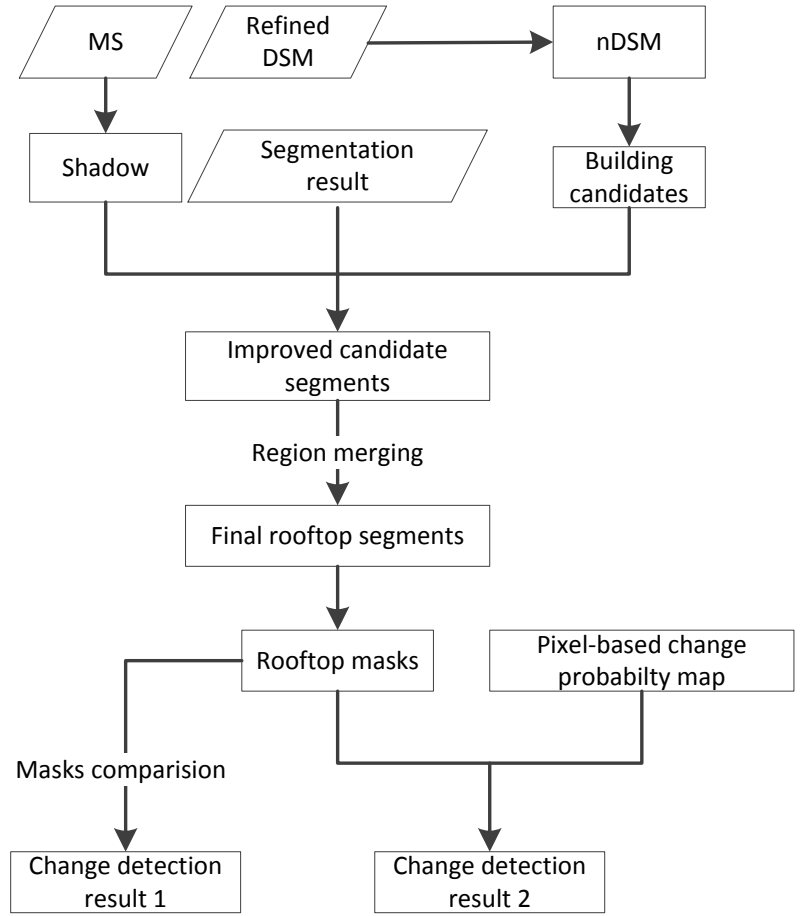

Figure 5. Workflow of rooftop extraction and change detection.

\subsubsection{Initial building candidate selection}

This step tries to find possible building locations with the help of the refined DSM. Therefore, the DSM is normalized with a morphological reconstruction function (Arefi and Hahn, 2005) to get the normalized DSM (nDSM). In the nDSM all pixels of ground regions have a value of less than about two meters, while objects like buildings have relative height values in reference to the ground. However, it is quite difficult to give a proper threshold value in this step. A higher threshold value leads to possible miss detections. When using a lower threshold, some buildings may be wrongly connected together due to limited DSM quality. Therefore, a low threshold value is used. After that shadows are adopted in this step to further separate the buildings. As shown in Figure 6, the buildings which are displayed in Figure 6 (a) as one mask are well separated in Figure 6 (b). After erosion, they are successfully split as six regions.

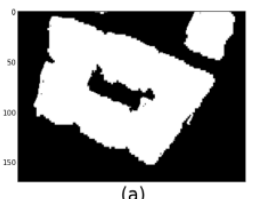

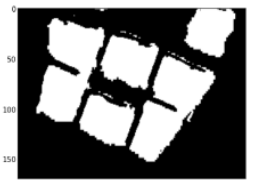

(b)

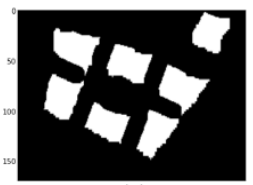

(c)
Figure 6. (a) Initial building candidate areas. (b) Building candidate areas after exclusion of shadow regions. (c) Building candidate areas after erosion.

\subsubsection{Building oriented region merging}

After the building locations have been labelled, these regions are dilated to recover the original region size. Then, the segments which are overlapped by these masks are selected as building candidate segments. All the segments with a size below a defined threshold are merged to their best fitting neighbours in terms of height and intensity. After the merging procedure, building detection is performed. In order to get the final building footprint map, a local threshold method is proposed. Instead of using a uniform threshold value for the whole test region, a minimum building height $T_{h, j}$ is automatically selected for each building candidate region (1 .. j) separately. For that, the average height $\mu H_{j}$ and the standard deviation $\sigma\left(H_{j}\right)$ are calculated from the nDSM for those areas that overlay the segments of the candidate region. $\omega$ is thereby a weight to adjust the threshold. Based on this, the local threshold is defined that distinguishes between buildings and background.

$T_{h, j}=\mu H_{j}-\omega * \sigma\left(H_{j}\right)$

This provides benefits especially when the previous separation procedures failed, and if there is more than one building within one building candidate region left, as is shown in Figure 7. In such cases it is possible to separate large regions into single building areas, as shown in Figure 7 (d). Therefore it is needed, that the areas between the buildings exhibit a lower average height than the buildings themselves (see Figure 7(c)).

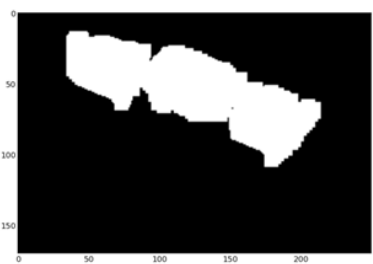

(a)

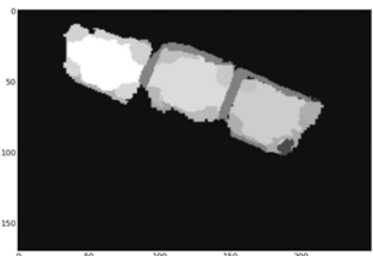

(c)

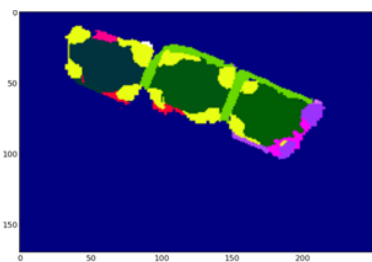

(b)

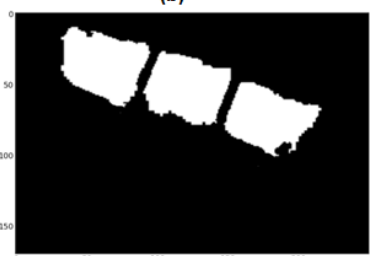

(d)
Figure 7. (a) Building candidate area. (b) Segments after merging. (c) Average height of the Segments. (d) Building footprint after applying the local threshold.

\subsubsection{Change detection}

After the building footprints are derived from the pre- and postevent data, building changes can be detected by comparing them on the object and pixel level. Each building footprint in the later scene that has a low overlap rate with one of the earlier scene, is supposed to be a new object. Therefore a minimum rate needs to be defined as the threshold in highlighting the changed object.

The other possibility to detect newly built buildings is to fuse the building footprint from the post-event data and the change indicator derived with the pixel-based change detection approach. In this approach, an existing pixel-based 3D change detection approach was used which was proposed by Tian et al (2015). Thereby, the building change probability of each pixel is determined by using a belief function model. After that the average change probability of each building mask can be calculated. Building masks with a higher probability to be changes remain in the change detection results.

\section{EXPERIMENT}

\subsection{Data sets}

Two test sites (a, b) were chosen for the experiments. Both are located in a mixed industrial and residential area in the east of Istanbul. This area is dynamic and fast changing, which makes it a good test region for change detection. In Figure 8, the images (a1, b1) are shown, which were captured on the 24th of August 2011 and (a2, b2) at the 7th of July 2012. They have a 
size of $350 \times 400 \mathrm{~m}$ (a) and $500 \times 500 \mathrm{~m}$ (b), respectively. All images are acquired by the WorldView- 2 satellite, which has one panchromatic band and eight multispectral bands. The multispectral bands are covering a spectral range from 400nm to $1050 \mathrm{~nm}$ and with a spatial resolution of $1.84 \mathrm{~m}$ (Padwick et al., 2010). Also along track panchromatic stereo data have been acquired in the same orbit.

The DSM was calculated from this panchromatic stereo data and has originally 1 meter resolution (Tian et al., 2013). The DSMs have been resampled to 0.5 meter resolution in order to have the same resolution as panchromatic images. After pansharpening the resolution of the multispectral image could be improved to $0.5 \mathrm{~m}$, the same as the resolution of the panchromatic image.

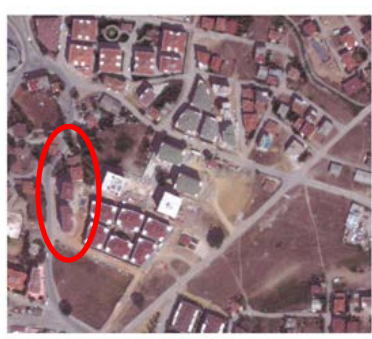

(a1)

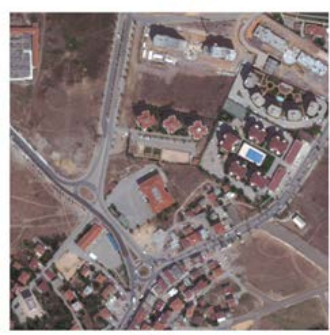

(b1)

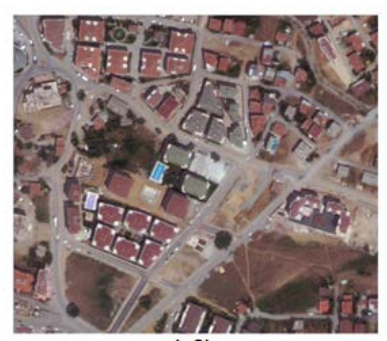

(a2)

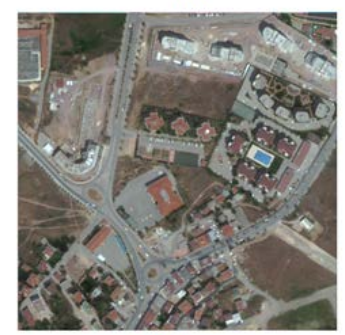

(b2)
Figure 8. Study sites: (a1, b1) are the pre-event images. (a2, b2) are the after-event change images.

\subsection{Results and evaluation}

\subsubsection{Building rooftop extraction}

In the original generated DSMs, the boundaries of objects are usually very blurry due to occlusions near the building boundaries leading to no-data areas close to buildings, which have been filled using interpolation. After the line based refinement, the boundaries of some buildings are sharper than before.
For the building candidate detection procedure, a threshold of 4 meter is set to select the high-level pixels. After the preprocessing procedure, as described in the methodology part, all segments within a candidate region are kept and then tried to merge. Thereby, at first all areas with a size smaller than 70 pixels $\left(17.5 \mathrm{~m}^{2}\right)$ are merged to their nearest neighbours, if the height difference between them is smaller than two meters. After that, merging is performed for all segments by using the multi feature merging procedure. Eq. (1) with $\omega=1.5$ is used to Eq. (1) to calculate the local threshold. All segments with an average height higher than the threshold are forming the building and are transferred into the building footprint.

The obtained building rooftop masks have been overlaid with the building reference map and are shown in Figure 9. It shows only the rooftop mask from the post-event data. In this result the majority of the large size buildings are green which represent true positive, while especially small buildings are often blue. This trend is also represented in Table 1. In Table 1, the result is evaluated for different building sizes. While only few buildings with a size smaller than $125 \mathrm{~m}^{2}$ are detected, almost all buildings larger than $375 \mathrm{~m}^{2}$ are detected. Very few false positive objects were thereby recognized. As Figure 9 shows, most detected building boundaries match well with the manually labelled reference data. The statistics are presented in Table 2, where the number of pixels for each of the classes can be seen, for all four test regions. The true positive, true negative, false positive, false negative detection rates and the Kappa Index of Agreement (KIA) (Tian et al., 2013) are calculated.

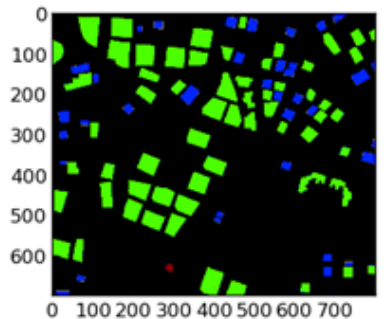

(a)

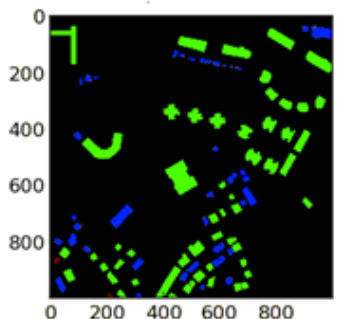

(b)
Figure 9. Detected building rooftop result on the post-event dataset (Green: True positive; Red: False positive; Blue: False negative).

\begin{tabular}{|c|c|c|c|c|c|c|c|c|c|}
\hline & \multicolumn{2}{|c|}{$\begin{array}{c}\text { building size }<125 \\
{\left[\mathrm{~m}^{2}\right]}\end{array}$} & \multicolumn{2}{c|}{$\begin{array}{c}125\left[\mathrm{~m}^{2}\right]<\text { building } \\
\text { size }<250\left[\mathrm{~m}^{2}\right]\end{array}$} & \multicolumn{2}{c|}{$\begin{array}{c}250\left[\mathrm{~m}^{2}\right]<\text { building } \\
\text { size }<375\left[\mathrm{~m}^{2}\right]\end{array}$} & \multicolumn{2}{c|}{$\begin{array}{c}\text { building size }>= \\
375\left[\mathrm{~m}^{2}\right]\end{array}$} & Over all \\
\cline { 2 - 9 } & True & $\begin{array}{c}\text { False } \\
\text { negative }\end{array}$ & True & $\begin{array}{c}\text { False } \\
\text { negative }\end{array}$ & True & $\begin{array}{c}\text { False } \\
\text { negative }\end{array}$ & True & $\begin{array}{c}\text { False } \\
\text { negative }\end{array}$ & $\begin{array}{c}\text { False } \\
\text { positive }\end{array}$ \\
\hline a1 & 1 & 20 & 21 & 10 & 11 & 4 & 19 & 0 & 0 \\
a2 & 7 & 24 & 20 & 9 & 11 & 2 & 9 & 0 & 1 \\
b1 & 4 & 17 & 19 & 17 & 9 & 2 & 15 & 4 & 2 \\
b2 & 9 & 30 & 18 & 9 & 8 & 1 & 11 & 2 & 2 \\
\hline
\end{tabular}

Table 1. Object based evaluation of the building footprints.

\begin{tabular}{|c|c|c|c|c|c|}
\hline & True positive & False negative & False positive & True negative & KIA \\
\hline a1 & 65434 & 30476 & 8218 & 455872 & 0.73 \\
a2 & 68818 & 24781 & 9385 & 457016 & 0.77 \\
b1 & 59355 & 46066 & 11198 & 883381 & 0.64 \\
b2 & 70056 & 32301 & 11282 & 885871 & 0.74 \\
\hline
\end{tabular}

Table 2. Pixel based building footprint evaluation. 


\subsubsection{Change detection}

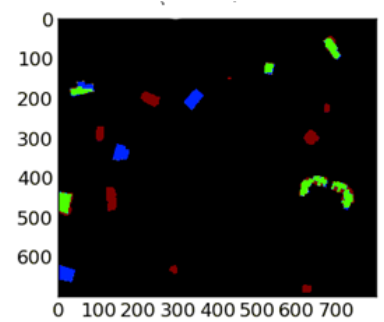

(a)

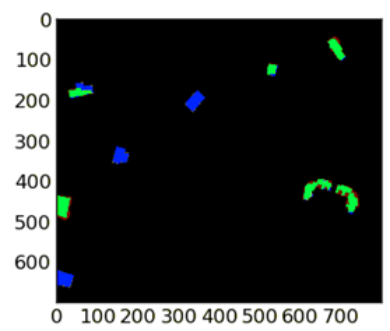

(c)

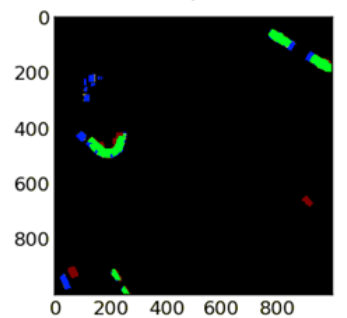

(b)

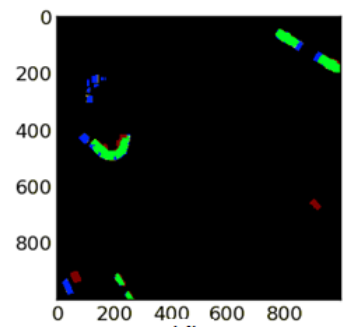

(d)

Figure 10. Change detection results, (a) and (b) are the region based change probability maps, (c) and (d) are the change detection results overlaid on the change reference mask (Green True positive; Red: False positive; Blue: False negative).

Figure 10(a) and 10(b) are the change detection results from post-classification approach, where the two building footprints of one scene are compared with each other. All Segments with an overlap ratio lower than 0.7 are considered to be newly built buildings. The change detection results are overlaid on the change reference data. The region/ pixel based building mask evaluation approaches are further used to evaluate the change detection results. It can be seen that again the most of the larger new buildings are detected, while especially smaller ones are missing. However, there is also a big number of false alarms (red objects) that is leading to smaller KIA values ( 0.51 for test site $a$ and 0.65 for Test site $b$, see Table 3). Better results are achieved with the second change detection approach (M2- $a$ and M2-b). As can be seen in Figure 10(c), much fewer false positive are observed. The KIA values have been improved to 0.66 and 0.71 , respectively. The region based evaluation results are displayed in Table 4.

Table 3. Pixel based evaluation of change detection results for the two test sites.

\begin{tabular}{|c|c|c|c|c|c|}
\hline & $\begin{array}{c}\text { True } \\
\text { positive }\end{array}$ & $\begin{array}{c}\text { False } \\
\text { negative }\end{array}$ & $\begin{array}{c}\text { False } \\
\text { positive }\end{array}$ & $\begin{array}{c}\text { True } \\
\text { negative }\end{array}$ & KIA \\
\hline$M 1-a$ & 6485 & 4653 & 7211 & 541651 & 0.51 \\
$M 1-b$ & 12136 & 6032 & 6415 & 975417 & 0.65 \\
$M 2-a$ & 6485 & 4653 & 1993 & 546869 & 0.66 \\
$M 2-b$ & 12136 & 6032 & 3840 & 977992 & 0.71 \\
\hline
\end{tabular}

Table 4. Region based evaluation of change detection results for the two test sites.

\begin{tabular}{|c|c|c|c|}
\hline & $\begin{array}{l}\text { True } \\
\text { positive }\end{array}$ & $\begin{array}{l}\text { False } \\
\text { negative }\end{array}$ & $\begin{array}{l}\text { False } \\
\text { positive }\end{array}$ \\
\hline$M 1-a$ & 6 & 3 & 8 \\
$M 1-b$ & 5 & 9 & 2 \\
$M 2-a$ & 6 & 3 & 0 \\
$M 2-b$ & 5 & 9 & 2 \\
\hline
\end{tabular}

\section{DISCUSSION}

The DSM refinement method is leading especially to a better distinction of buildings that are standing close together. Further, single objects are getting a more homogeneous height value. Although the improvements are limited, they are bringing benefits for the following segmentation and are by this improving the final results. Segmentation without the refinement would be also possible, but the results would be more vulnerable to errors.

Over-segmentation could be handled with merging, undersegmentation would be more difficult to refine. Therefore, the first segmentation results are over-segmented and then refined with a merging procedure. For this, it is important to have a first segmentation that match well with the object boundaries. For boundaries that are not well recognizable in some of the images, multiple input features are used. Although this leads to longer processing times, sometimes it is necessary for accurate results. To reduce over-segmentation effects, merging is performed for the whole image, although just the building areas are of interest.

In this approach, all segments with a height larger than the local height based threshold are assumed to be buildings. Thus, it is possible to detect and separate multiple buildings within one candidate area. However, sometimes there are parts of the buildings missing, like is shown in the red marked area in Figure 10. This is due to height differences within buildings and due to the already discussed cases of over-segmentation in buildings, which cannot be fully eliminated. Other approaches, like (Lee et al. 2003) and (Tian and Reinartz, 2013) propose to use Hough lines to extract accurate building shapes. Hough lines are thereby representing the main directions in an image. In case of having just one, or a few quadrangle buildings within one candidate area, this might truly increase the accuracy of the building footprints. Having many buildings in one candidate area, might however decrease the accuracy.

Additional to these method based errors, some buildings could not be detected due to errors in the underlying data. Especially the inaccuracy of the DSM is causing some missing buildings, as objects need to have a certain height in order to be determined as a building. Overall, it can be stated that it is possible with this method to detect the majority of the big buildings, while small buildings are not well recognized. Due to the use of the previous segmentation, the shapes of the building footprints are close to those of the real buildings. Sometimes however, small parts of the buildings are missing, which influences the final result accuracy.

\section{CONCLUSION}

For urban planning, map updates, insurance, hazard prevention and real estate monitoring it is important to have actual data about all buildings in an area. Especially in urban areas, where the façades, the structures and alignment of buildings change fast, it is important to update data efficiently and reliably; therefore, there is a growing interest in the development of tools and sensors used for the detection of buildings - with reduced working times and reasonable costs (Gamba and Houshmand, 2000).

In this paper, an efficiently automatic building rooftop extraction and building change detection approach is shown and accurate results can be achieved based on WorldView-2 stereo data. It was shown that the results are very dependent on the DSMs quality. Better results would be achieved when more 
accurate DSMs are available. Together with a further improved method this could lead to better usability of the automatic building footprints and change detection results. As lot of buildings/changes are correctly extracted with the proposed approach, it can be used to get a fast overview over an area. For especially smaller buildings are sometimes missing and the building boundaries are not always accurate, it should however be used in a semi-automatic way together with visual inspection to update maps or databases. In this research, fusing of the rooftop mask and the decision probability map has delivered the change detection results with higher accuracy. However, if there would be already one building footprint available, for instance some GIS cadastral data etc., the post-classification approach could be even more efficient, to detect change objects. Further comparison with existing approaches will be performed.

\section{REFERENCES}

Arefi, H. and Hahn, M., 2005. A morphological reconstruction algorithm for separating off-terrain points from terrain points in laser scanning data. International Archives of Photogrammetry, Remote Sensing and Spatial Information Sciences, 36(3/W19), pp.120-125.

Comaniciu, D. and Meer, P., 2002. Mean shift: A robust approach toward feature space analysis. IEEE Transactions on pattern analysis and machine intelligence, 24(5), pp.603-619.

Coppin, P., Jonckheere, I., Nackaerts, K., Muys, B. and Lambin, E., 2004. Review ArticleDigital change detection methods in ecosystem monitoring: a review. International journal of remote sensing, 25(9), pp.1565-1596.

Gamba, P. and Houshmand, B., 2000. Digital surface models and building extraction: A comparison of IFSAR and LIDAR data. IEEE Transactions on Geoscience and Remote Sensing, 38(4), pp.1959-1968.

Liu, Z.G., Mercier, G., Dezert, J. and Pan, Q., 2014. Change detection in heterogeneous remote sensing images based on multidimensional evidential reasoning. IEEE Geoscience and Remote Sensing Letters, 11(1), pp.168-172.

Lu, D., Mausel, P., Brondizio, E. and Moran, E., 2004. Change detection techniques. International journal of remote sensing, 25(12), pp.2365-2401.

Padwick, C., Deskevich, M., Pacifici, F. and Smallwood, S., 2010, April. WorldView-2 pan-sharpening. In Proceedings of the ASPRS 2010 Annual Conference, San Diego, CA, USA (Vol. 2630).

Petit, C., Scudder, T. and Lambin, E., 2001. Quantifying processes of land-cover change by remote sensing: resettlement and rapid land-cover changes in south-eastern Zambia. International Journal of Remote Sensing, 22(17), pp.3435-3456

Qin, R., Tian, J. and Reinartz, P., 2016. 3D change detectionapproaches and applications. ISPRS Journal of Photogrammetry and Remote Sensing, 122, pp.41-56.

Singh, A., 1989. Digital change detection techniques using remotelyWsensed data. International Journal of Remote Sensing, 10(6), p.989L1003.

Tian, J., 2013. 3D change detection from high and very high resolution satellite stereo imagery (Doctoral dissertation, Universität Osnabrück).

Tian, J., Reinartz, P., d'Angelo, P. and Ehlers, M., 2013. Region-based automatic building and forest change detection on
Cartosat-1 stereo imagery. ISPRS Journal of Photogrammetry and Remote Sensing, 79, pp.226-239.

Tian, J. and Reinartz, P., 2013. Fusion of multi-spectral bands and DSM from Worldview-2 Stereo imagery for building extraction. In Proceedings of 2013 Joint Urban Remote Sensing Event (JURSE), pp. 135-138.

Tian, J., Dezert, J. and Reinartz, P., 2015. Refined building change detection in satellite stereo imagery based on belief functions and reliabilities. In 2015 IEEE International Conference on Multisensor Fusion and Integration for Intelligent Systems (MFI), pp. 160-165. 